\title{
LOW VELOCITY \\ GRAVITATIONAL CAPTURE BY LONG COSMIC STRINGS *
}

\author{
Don N. Page ${ }^{\dagger}$ \\ CIAR Cosmology Program, Institute for Theoretical Physics \\ Department of Physics, University of Alberta \\ Edmonton, Alberta, Canada T6G 2J1
}

(1999 February 11)

\begin{abstract}
Coupled ordinary differential equations are derived for the distant gravitational interaction of a compact object of mass $M$ and charge $Q$ with an initially straight, infinitely long, cosmic string of tension $\mu \ll 1 / G \equiv 1$, when the relative velocities are very low compared to the speed of light $c \equiv 1$. (An intermediate result of this derivation is that any localized force $\mathbf{F}(t)$ on the string that is confined to a single plane perpendicular to the initial string configuration gives the intersection of the string with this plane - the point where the force is applied - the velocity $\mathbf{F}(t) /(2 \mu)$.) The coupled equations are then used to calculate the critical impact parameter $b_{\text {crit }}\left(v_{0}\right)$ for marginal gravitational capture as a function of the incident velocity $v_{0}$. For $v_{0} \ll\left(1-Q^{2} / M^{2}\right)^{1 / 3} \mu^{2 / 3}$, so that the string acts relatively stiffly,

$b_{\text {crit }} \approx \frac{\pi}{4}\left[12 \mu^{3}\left(1-\frac{Q^{2}}{M^{2}}\right)^{4}\right]^{\frac{1}{5}} M v_{0}^{-\frac{7}{5}}+\frac{\pi}{5}\left[\frac{27}{16 \mu}\left(1-\frac{Q^{2}}{M^{2}}\right)^{2}\right]^{\frac{1}{5}} M v_{0}^{-\frac{1}{5}}+O\left(\frac{M v_{0}}{\mu}\right)$. For $\left(1-Q^{2} / M^{2}\right)^{1 / 3} \mu^{2 / 3} \ll v_{0} \ll 1-Q^{2} / M^{2}$, so that the string acts essentially as a test string that stays nearly straight,

$b_{\text {crit }} \approx\left[\frac{\pi}{2}\left(1-\frac{Q^{2}}{M^{2}}\right)\right]^{\frac{1}{2}} M v_{0}^{-\frac{1}{2}}+\frac{\pi}{4}\left(1-\frac{Q^{2}}{M^{2}}\right) \mu M v_{0}^{-2}+O\left(M \mu^{2} v_{0}^{-\frac{7}{2}}\right)+O(M)$.

Between these two limits the critical impact parameter is found numerically to fit a simple algebraic combination of these two formulas to better than $99.5 \%$ accuracy.
\end{abstract}

${ }^{*}$ Alberta-Thy-01-99, gr-qc/9902038

†Internet address: don@phys.ualberta.ca 


\section{Introduction}

The gravitational interactions between compact objects and long cosmic strings have been calculated in several recent papers [1, 2, 3, 4, in the test-string approximation, in which one assumes that the dimensionless string tension $\mu$ (using units in which Newton's gravitational constant is $G=1$ ) is much smaller than any other parameter whose ratio with $\mu$ is relevant. Then the string obeys the Nambu-Goto equations of motion [5, 6, 4] in the gravitational field of the object and has negligible back reaction on the motion of the object.

It is indeed a good approximation for cosmic strings that $\mu \ll 1$, but I shall show that at low initial relative velocities $v_{0}$ and near the critical impact parameter, the ratio $\mu / v_{0}^{3 / 2}$ is important, so the test-string approximation is accurate only if the velocity is large in comparison to $\mu^{2 / 3}$. For velocities very low compared with this, the string acts relatively stiffly, so that the motion of the object responds more than the motion of the string.

Here I shall derive coupled ordinary differential equations of motion for an initially straight, infinitely long, cosmic string interacting gravitationally with a compact object of mass $M$ and charge $Q$ (i.e., one whose size is negligible in comparison with the distance between the object and the string), using the approximations $\mu \ll 1$ and $v_{0} \ll 1$ but allowing $\mu / v_{0}^{3 / 2}$ to be arbitrary. The low velocity allows the part of the string nearest the object to remain nearly straight, and then the force $\mathbf{F}$ on the string is a simple function of the string-object separation and relative velocity. On a much larger scale this force is nearly localized and hence gives the point on the string nearest the object the velocity $\mathbf{F} /(2 \mu)$, as we shall see.

For fixed $M, Q$, and $\mu$, after taking out Poincare transformations there is a two-parameter set of solutions that can be characterized by the initial speed $v_{0}$ of the object relative to the string (when they are infinitely far away in the infinite past) and by the impact parameter $b$ (how far the object would miss the string if both moved uniformly with no interactions). For each $v_{0}$ there is a critical impact parameter, $b_{\text {crit }}$, such that if $b<b_{\text {crit }}$ the object becomes gravitationally captured and bound to the string, but if $b>b_{\text {crit }}$, the object and string scatter and asymptotically move freely of each other. In this paper the coupled equations of motion of the object and the string will be solved to give the function $b_{\text {crit }}\left(v_{0}\right)$, analytically when the ratio $\mu / v_{0}^{3 / 2}$ is either very large or very small, and numerically otherwise. In the latter case simple algebraic combinations of the analytic results at the two extremes reproduce the numerical results over the entire range to within $1 \%$, or even within $0.43 \%$ if two arbitrary exponents are chosen appropriately. 


\section{Gravitational force between an object and a straight string}

Strictly speaking, the gravitational interaction between an object and a string should be done in terms of curved spacetime. However, when $\mu \ll 1$ and when one is in the weak-field regime of the object (which is where the string is, under the assumption that the object size is much smaller than its distance to the string), one can do an analysis in terms of fictitious forces acting in a fictitious flat background spacetime, just as one does for Newtonian gravity (though one need not assume that the gravitational field of the string is Newtonian or that the object velocity is low to do this).

We assume that the string is infinitely long and is nearly straight in the region where it is fairly near its minimum separation from the object, with no oscillations coming in along the string from infinity. Given the assumed initial straight, static configuration of the string, this assumption of approximate straightness of the part of the string nearest the object for all times is justified by the assumption of low transverse velocities, since the fact that the disturbances of the string propagate up and down it, away from their source, at the speed of light, means that the length of string that is disturbed is much greater than the transverse disturbance, so that the angular bending of the string is small, even if the linear bending becomes large.

Then we can go to the frame in which the part of the string nearest the object is at rest along the $z$-axis and the object is in the $x$-y plane with velocity of magnitude $v_{r}$ in the positive $x$-direction, the relative velocity of the object with respect to the piece of string nearest the object. To linear order in $M$ and $Q$, the gravitational force of the string on the object is zero when $v_{r}=0$. However, the string produces a conical spacetime with a deficit angle $2 \pi(4 \mu)$ [8, so when this is flattened out in the fictitious flat background spacetime with azimuthal angle $\varphi$ that ranges from 0 to $2 \pi$, a moving object appears to be bending toward the string at the rate of $4 \mu$ times the rate at which $\varphi$ is changing, i.e., at the rate of $4 \mu \dot{\varphi}=4 \mu v_{r} y / r^{2}$, where

$$
r=\sqrt{x^{2}+y^{2}}
$$

is the distance from the object to the string (ignoring relative corrections of order $\mu$ that depend on how the conical spacetime is flattened out to produce the fictitious flat spacetime).

When this bending rate is multiplied by the momentum $p=M \gamma_{r} v_{r}$ of the object, where $\gamma_{r}=1 / \sqrt{1-v_{r}^{2}}$, one gets the part of the transverse fictitious force on the object that is linear in $M, 4 \mu M \gamma_{r} v_{r}^{2} y / r^{2}$. The longitudinal fictitious force (in the direction of the object velocity $\mathbf{v}_{r}$ in the instantaneous frame of the piece of string closest to the object, using boldface symbols to denote spatial vectors in the twodimensional plane perpendicular to the string in the fictitious flat spacetime) that is linear in $M$ depends on the flattening procedure used to generate the fictitious 
flat metric. One can choose it so that the total fictitious force on the object that is linear in $M$ is

$$
\mathbf{F}_{\text {linear }}=-4 \mu M \gamma_{r} v_{r}^{2} \mathbf{r} / r^{2} .
$$

By Newton's third law or conservation of momentum, there will be an equal and opposite fictitious force on the string to describe its motion in the fictitious flat spacetime.

To explain and justify this fictitious force in greater detail, consider the geodesic motion of a test mass $M$ in the conical spacetime of a cosmic string [8],

$$
\begin{aligned}
d s^{2} & =-d t^{2}+d z^{2}+d \rho^{2}+(1-4 \mu)^{2} \rho^{2} d \varphi^{2} \\
& =-d t^{2}+d z^{2}+r^{-8 \mu}\left(d r^{2}+r^{2} d \varphi^{2}\right)=-d t^{2}+d z^{2}+e^{2 \phi}\left(d r^{2}+r^{2} d \varphi^{2}\right),
\end{aligned}
$$

where

$$
r=[(1-4 \mu) \rho]^{1 /(1-4 \mu)}
$$

and

$$
\phi=-4 \mu \ln r
$$

(not to be confused with the azimuthal angle $\varphi$ ).

One can go to the frame in which the object moves in the plane $z=0$, in which case the motion is in an ultrastatic $(2+1)$-dimensional spacetime with conformally flat spatial sections, whose general metric form is

$$
d s^{2}=-d t^{2}+e^{2 \phi} \delta_{i j} d x^{i} d x^{j} .
$$

Timelike geodesics of such metrics have constant

$$
E=M \frac{d t}{d \tau}
$$

and constant

$$
P^{2}=M^{2} e^{2 \phi} \delta_{i j} \frac{d x^{i}}{d \tau} \frac{d x^{j}}{d \tau}=E^{2}-M^{2}
$$

One can also readily show that the spacetime geodesic equation implies that the spatial trajectory is itself a geodesic of the spatial metric $e^{2 \phi} \delta_{i j} d x^{i} d x^{j}$ and obeys the equation

$$
\frac{d^{2} x^{i}}{d \sigma^{2}}=\left(\delta^{i j}-\frac{d x^{i}}{d \sigma} \frac{d x^{j}}{d \sigma}\right) \phi_{, j},
$$

where $\sigma$ is the spatial distance along the trajectory,

$$
d \sigma^{2}=\delta_{i j} d x^{i} d x^{j}
$$

in the fictitious flat spacetime metric

$$
d s_{b}^{2}=-d t_{b}^{2}+\delta_{i j} d x^{i} d x^{j} .
$$


One can reproduce the spatial geodesic of the conformally-flat spatial metric $e^{2 \phi} \delta_{i j} d x^{i} d x^{j}$ by a fictitious spatial force

$$
\mathbf{F}=\frac{d \mathbf{p}}{d t_{b}}=M \gamma_{r} v_{r}^{2} \nabla \phi+f \mathbf{v}_{r}
$$

in the fictitious flat spacetime, where

$$
\mathbf{p}=M \gamma_{r} \mathbf{v}_{r}=M \gamma_{r} d \mathbf{r} / d t_{b}
$$

is the fictitious flat spacetime momentum of the particle of mass $M$, of flat spacetime position vector $\mathbf{r}$ (with components $x^{i}$ that are raised and lowered by the fictitious flat spatial metric $\delta_{i j} d x^{i} d x^{j}$ in this fictitious force analysis), of spatial velocity $\mathbf{v}_{r}=$ $d \mathbf{r} / d t_{\mathrm{b}}$ with magnitude $v_{r}=\sqrt{\mathbf{v}_{r} \cdot \mathbf{v}_{r}}=\sqrt{\delta_{i j} v_{r}^{i} v_{r}^{j}}$, and of $\gamma_{r}=1 / \sqrt{1-v_{r}^{2}}$.

In the expression above for the fictitious force $\mathbf{F}$, one can have an arbitrary function $f$ multiplying the velocity $\mathbf{v}_{r}$ if one just wants to reproduce the spatial trajectory of the geodesic of the actual metric (6). If one also wants to reproduce the time dependence of the trajectory, so $x^{i}\left(t_{b}\right)$ under the fictitious force in the fictitious metric has the same dependence on $t_{b}$ as $x^{i}(t)$ does on $t$ for the geodesic in the actual metric, then one needs

$$
f=-M \gamma_{r}\left(\gamma_{r}^{2}+1\right) \mathbf{v}_{r} \cdot \nabla \phi
$$

However, for the purposes of this paper, the precise time dependence of the trajectory of an object passing a cosmic string is not so important as the trajectory itself, so I shall set $f=0$. Then if one uses the form of $\phi$ given by Eq. (5) for the cosmic string, one gets the fictitious force given by Eq. (2).

To quadratic order in $M$ and $Q$, A. G. Smith [9] calculated from the effect of the deficit angle on the three-dimensional Laplacian for the Newtonian gravitational and electrostatic fields of the object that the string exerts an attractive force (if $\left.M^{2}>Q^{2}\right)$ of magnitude $\pi \mu\left(M^{2}-Q^{2}\right) /\left(4 r^{2}\right)$ on the object when the relative velocity $v_{r}$ can be neglected. (When $v_{r}$ is not negligible in comparison with unity, the quadratic force is overwhelmed by the linear force, so one does not need the highvelocity corrections to the quadratic force.) In two-dimensional vectorial form,

$$
\mathbf{F}_{\text {quadratic }}=-\pi \mu\left(M^{2}-Q^{2}\right) \mathbf{r} /\left(4 r^{3}\right) \text {. }
$$

The conservation of momentum implies that the object exerts an equal but opposite force on the string, and this indeed gives precisely the bending of a distant static string in the Reissner-Nordstrom metric [10].

When we add the fictitious forces that are linear and quadratic in $M$ and $Q$, we get a total fictitious force on the object that, in the limit of a small dimensionless string tension $\mu \ll 1$ and in the limit of a large separation $r \gg M$, is approximately

$$
\mathbf{F}_{M}=-\frac{4 \mu M \gamma_{r} v_{r}^{2} \mathbf{r}}{r^{2}}-\frac{\pi \mu\left(M^{2}-Q^{2}\right) \mathbf{r}}{4 r^{3}}
$$


The first term is valid for all velocities $\mathbf{v}_{r}$ but neglects additional terms in the direction of $\mathbf{v}_{r}$, and the second term, which is only important if the velocity is very small, neglects corrections when $v_{r}$ is not small.

\section{Motion of a long string with a localized force}

In the fictitious flat spacetime, the response of the compact object to the fictitious force is simply the relativistic version of Newton's second law, the first equality of

Eq. (12). However, the response of the string to the equal and opposite fictitious force needs to be worked out.

As noted above, the low-transverse-velocity assumption means that the length of string that is bent (which develops at the speed of light) is much greater than the transverse bending (which develops at the transverse velocity of the string, much less than the speed of light by assumption). Hence, one can look at the bending on a scale much larger than that of the transverse separation between the string and the object and yet much smaller than that of the length of string that is bent. On this scale, the force on the string appears to be localized at the position on the string nearest the object and is given to good approximation by Eq. (16) in terms of the string-object transverse separation $\mathbf{r}$ and the relative velocity $v_{r}$.

Let us do a general analysis of the effect of such a localized force on an initially static, straight cosmic string, without, in the general analysis, assuming that the transverse velocity of the string is small, even though in our particular application we need this assumption to justify our assumption that the force is effectively local and is given by Eq. (16).

Suppose a time-dependent horizontal force in the $x-y$ plane is applied at $z=0$ to an infinite string initially lying at rest along the $z$-axis (vertically), with no other forces on it. This force causes equal horizontal disturbances to go up (for $z>0$ ) and down (for $z<0$ ) the string at the speed of light (propagating away from where they are generated by the time-dependent force, at $z=0$; assuming that the infinite string was initially at rest and has no forces on it at $z \neq 0$ implies that no disturbances are coming in to $z=0)$ :

$$
\mathbf{r}_{s}(t, z)=\theta(z) \mathbf{r}_{\perp}(t-z)+\theta(-z) \mathbf{r}_{\perp}(t+z)
$$

where $\theta(z)$ is the Heaviside step function of $z$ and each of the two occurrences of $\mathbf{r}_{\perp}$ represents the same function of the argument inside the following bracket (either $t-z$ for the first term, which is nonzero for $z>0$ where $\theta(z)=1$ and $\theta(-z)=0$, or $t+z$ for the second term, which is nonzero for $z<0$ where $\theta(-z)=1$ and $\theta(z)=0)$.

Focus on the infinitesimal piece of string just above $z=0$ at some arbitrary time $t$. Its tilt or slope away from vertical is

$$
\frac{\partial \mathbf{r}_{s}(t, z)}{\partial z}=-\frac{\partial \mathbf{r}_{s}(t, z)}{\partial t}=-\dot{\mathbf{r}}_{\perp}
$$


where the overdot in the last term denotes a derivative with respect to the unlisted argument $t-z$ evaluated at $z=0$. Denote the magnitude of this slope by

$$
v_{s} \equiv\left|\dot{\mathbf{r}}_{\perp}\right| \equiv \sqrt{\dot{\mathbf{r}}_{\perp} \cdot \dot{\mathbf{r}}_{\perp}}=\tan \psi
$$

where $\psi$ is the instantaneous angle of the tilt from the vertical.

This piece of string can be considered to be moving straight upward at the speed of light (unity), the velocity at which the pattern moves for $z>0$, but locally any component of the velocity parallel to the string has no effect. To calculate the energy and momentum carried by a piece of string, what is relevant is the transverse velocity, the component perpendicular to the string. Here the infinitesimal piece of string is tilted at an angle $\psi$ from the vertical, so the transverse velocity is tilted at an angle $\pi / 2-\psi$ from the vertical direction along which the pattern speed is unity. Therefore, the magnitude of the transverse velocity is

$$
v_{t}=\sin \psi=\frac{v_{s}}{\sqrt{1+v_{s}^{2}}},
$$

and the associated relativistic $\gamma$ factor is

$$
\gamma_{t}=\frac{1}{\sqrt{1-v_{t}^{2}}}=\sec \psi=\sqrt{1+v_{s}^{2}}
$$

The length of this infinitesimal piece of string is

$$
d l=(\sec \psi) d z=\sqrt{1+v_{s}^{2}} d z
$$

and its energy is

$$
d E=\mu \gamma_{t} d l=\mu\left(\sec ^{2} \psi\right) d z=\mu\left(1+v_{s}^{2}\right) d z .
$$

The transverse momentum (at the angle $\psi$ from the horizontal $x$ - $y$ plane) is the transverse velocity times the energy, with magnitude

$$
d p_{t}=v_{t} d E=\mu\left(\sin \psi \sec ^{2} \psi\right) d z=\mu v_{s} \sqrt{1+v_{s}^{2}} d z .
$$

The vertical component of this momentum is

$$
d p_{z}=\sin \psi d p_{t}=\mu\left(\tan ^{2} \psi\right) d z=\mu v_{s}^{2} d z
$$

and the horizontal component has magnitude

$$
d p_{h}=\cos \psi d p_{t}=\mu(\tan \psi) d z=\mu v_{s} d z
$$

and is in the direction of $\dot{\mathbf{r}}_{\perp}$. 
This infinitesimal piece of the displaced-string pattern that is moving upward at the speed of light from the force at $z=0$ takes a time $d t=d z$ to be generated. A mirror-image pattern is moving downward at the speed of light from the force at $z=0$, and during the same infinitesimal time an infinitesimal piece of string with the same magnitude of $d z$ is generated moving downward just below $z=0$. The vertical components of these two pieces are opposite and hence cancel, but the horizontal components are in the same direction and so add to make the two infinitesimal pieces of string generated during the time $d t$ have a total momentum of

$$
d \mathbf{p}=2 \mu \dot{\mathbf{r}}_{\perp} d t=2 \mu d \mathbf{r}_{\perp}=2 \mu d \mathbf{r}_{s},
$$

where here and henceforth I shall use $\mathbf{r}_{s}$ for $\mathbf{r}_{s}(t, 0)$ at $z=0$, the horizontal position of the string in the $x-y$ plane where the force is applied.

Since the string was assumed to start off from rest along the $z$-axis, at $\mathbf{r}_{s}(t, z)=0$ for large negative $t$, the total momentum of the string is simply

$$
\mathbf{p}_{s}=2 \mu \mathbf{r}_{s},
$$

and the force on the string is

$$
\mathbf{F}_{s}=\frac{d \mathbf{p}_{s}}{d t}=2 \mu \dot{\mathbf{r}}_{s} .
$$

The string will react back with an equal and opposite force on whatever is forcing it to move horizontally. It is interesting that this reaction force is precisely an idealized friction force directly proportional to velocity. The fact that momentum is carried away along the string at the speed of light, never to return (since the string is assumed to be infinite), is apparently what allows an initially straight, infinitely long string to act as a perfect source of friction.

One application of Eq. (28) is to give a very simple derivation of the net displacement of an initially straight string by a gravitating object of mass $M$ that passes by with large impact parameter $b \gg M / v_{0}^{2}$, so that the fictitious force term linear in $M$, the first term of Eq. (16), dominates over the force term quadratic in $M$ and $Q$, the second term of Eq. (16). Then the object trajectory bends by half the deficit angle, i.e., by an angle $4 \pi \mu \ll 1$, so its momentum changes by

$$
\Delta p=4 \pi \mu p=4 \pi \mu M \gamma_{0} v_{0} .
$$

By Newton's third law, the string's momentum changes by the opposite amount, so Eq. (28) implies that the string gets displaced by

$$
\Delta r=\Delta p /(2 \mu)=2 \pi M \gamma_{0} v_{0},
$$

just as De Villiers and Frolov found [2]. 
If one inverts Eq. (29) to get

$$
\dot{\mathbf{r}}_{s}=\frac{\mathbf{F}_{s}}{2 \mu}
$$

one notices the curious fact that apparently a force larger than twice the string tension $\mu$ can move a string faster than the speed of light, seemingly a violation of special relativity, despite the fact that a completely (special) relativistic analysis was used in the derivation of the relationship between horizontal force and velocity. However, one can make two comments about this paradox:

First, for all finite horizontal speeds $v_{s}$ of the string, even 'superluminal' $v_{s}>1$, the physical transverse speeds $v_{t}$ of the string, given by Eq. (20) above, are less than the speed of light. The horizontal speed $v_{s}$ of the kink in the string at $z=0$ where the force is applied is somewhat analogous to the speed of the point where the two blades of a pair of scissors intersect. Even though the two blades are constrained to move slower than the speed of light, there is nothing in special relativity that constrains the intersection point to move slower than light.

One might object that the scissors are more nearly analogous to a pair of intersecting strings both moving in the same plane, whose intersection point can indeed move faster than light (at least if one assumes no interactions at the intersection, so that the two strings each move freely). In this case it seems to be the prior existence of each of the two strings on both sides of the intersection that allows the intersection to move faster than light while neither string does so. However, for the single string being considered in this paper, whose kink at $z=0$ moves horizontally faster than light if the force is greater than twice the string tension, there is no pre-existing string beyond the kink, so it seems that something physical must be moving faster than light.

The partial answer to this worry is that for a horizontal force greater than $2 \mu$, string is being created faster than light, but it is not being moved faster than light (in the transverse direction, the only motion that has a physical meaning for a longitudinal-boost-invariant string of the type being considered here). This is somewhat analogous to the possible nearly-instantaneous freezing of a lake, in which the water-ice boundary can in principle move faster than the speed of light as ice is created (but not moved) faster than light.

Another analogy would be electron-positron pair creation in a hypothetical imploding cylindrical electromagnetic wave that builds up a huge longitudinal electric field, say between two capacitor plates at the ends of the cylinder. The pair creation can in principle discharge the plates much faster than the time taken for light to go from one plate to the other. If one draws the Feynman diagrams of the pair creation and annihilation that accomplish this process, with positrons pictured as electrons moving backward in time, one can get electron lines that zigzag forward and backward between pair creations and annihilations and have a net spatial motion faster than light between the two plates. One might call this a tachyonic lightning bolt. And yet there would be no violation of special relativity with signals traveling faster 
than light in this hypothetical process, since it would be the imploding electromagnetic wave that locally causes the process, with no causes traveling faster than the speed of light.

Considering the creation of string faster than the speed of light leads on to the second comment, which is that although a string with a kink having a horizontal velocity $v_{s}>1$ does not seem to violate the principles of causality in special relativity (signals not traveling faster than light), it does seem to be associated with an instability analogous to tachyonic behavior. The example of the nearly-instantaneous freezing of a lake illustrates the instability of the liquid phase of water at low temperatures, and the example of the pair creation in the imploding electromagnetic field illustrates the instability of the zero-current 'vacuum' of the Dirac electron-positron field in the presence of a strong electric field. Similarly, tachyonic negative masssquared terms in relativistic wave equations, such as the Klein-Gordon equation, do not lead to violations of causality (since disturbances do not propagate faster than light whatever the sign of the mass-squared term) but instead lead to instabilities of exponential growth.

In the case of applying a strong force to a string, this can lead to an instability in the production of strings. For example, suppose the force is that of a gauge field on a massless charged particle attached to the string at the kink at $z=0$. (If the particle were massive, its inertia would prevent the kink from moving faster than light, so I shall assume the contrary to continue the argument.) But then if the force were greater than $\mu$, the energy that a pair of oppositely charged massless particles extract from the gauge field in being created and separated would be greater than the energy needed to create the string joining them. This would lead to the rapid pair production of pieces of string with these oppositely charged gauge particles at the ends in the strong gauge field, an instability that would presumably rapidly discharge the gauge field until the force on a charged particle would be no greater than $\mu$.

Therefore, if the force is that of a stable gauge field applied to a massless charged particle on the string, it appears that the maximum force is the string tension $\mu$, and therefore the maximum horizontal velocity of the string kink where the particle is attached is one-half the speed of light (relative to the distant parts of the string that are still at rest along the $z$-axis according to the assumption that the entire string was originally at rest along the $z$-axis and that the force is applied only at $z=0$ ). 


\section{Coupled motion of a slowly moving compact ob- ject and a nearly straight infinitely long string}

Now let us combine the expression (16) for the force between a compact object and a distant nearly straight long string with Newton's second law for the response of the object and with Eq. (32) for the response of the string, when the relative velocities are all small compared with the speed of light, so that Eq. (16) indeed applies for giving the force and so that the force is effectively localized (relative to the length of string that is bent) in order for Eq. (32) to apply as well. Use a fictitious flat spacetime metric with Cartesian coordinates chosen so that the string is initially at rest along the $z$-axis and the compact object moves in the $x$ - $y$ plane $(z=0)$, and use boldface letters to denote vectors in this plane. (The same letters when not bold will denote the magnitudes of the corresponding boldface vectors.) In particular, let $\mathbf{r}_{M}$ denote the position of the compact object (with mass $M$ and charge $Q$ ) relative to the origin $(x, y)=(0,0)$, let $\mathbf{r}_{s}$ denote the position of the string at $z=0$ relative to the origin, and let

$$
\mathbf{r}=\mathbf{r}_{M}-\mathbf{r}_{s}
$$

denote the position of the compact object relative to the string.

Then the nonrelativistic coupled equations of motion for the compact object and the string become

$$
M \ddot{\mathbf{r}}_{M}=-2 \mu \dot{\mathbf{r}}_{s}=-\frac{\pi \mu\left(M^{2}-Q^{2}\right) \mathbf{r}}{4 r^{3}}-\frac{4 \mu M(\dot{\mathbf{r}} \cdot \dot{\mathbf{r}}) \mathbf{r}}{r^{2}}
$$

It is now convenient to use the unit of time

$$
T_{u} \equiv \frac{M}{2 \mu}
$$

and the unit of length

$$
L_{u} \equiv\left[\frac{\pi}{16 \mu}\left(1-\frac{Q^{2}}{M^{2}}\right)\right]^{1 / 3} M,
$$

which we henceforth define to be unity (unless otherwise specified). In terms of these units the speed of light is no longer unity but is

$$
c=\left[\frac{\pi}{2} \mu^{2}\left(1-\frac{Q^{2}}{M^{2}}\right)\right]^{-1 / 3} \frac{L_{u}}{T_{u}}=\left[\frac{\pi}{2} \mu^{2}\left(1-\frac{Q^{2}}{M^{2}}\right)\right]^{-1 / 3} \gg 1 .
$$

Setting $T_{u}=L_{u}=1$, Eqs. (34) become

$$
\ddot{\mathbf{r}}_{M}=-\dot{\mathbf{r}}_{s}=-\frac{\mathbf{r}}{r^{3}}-\frac{4 \mu(\dot{\mathbf{r}} \cdot \dot{\mathbf{r}}) \mathbf{r}}{r^{2}} .
$$


At an arbitrary time $t_{i}$, the freely specifiable initial conditions are $\mathbf{r}_{s}\left(t_{i}\right), \mathbf{r}_{M}\left(t_{i}\right)$, and $\dot{\mathbf{r}}_{M}\left(t_{i}\right)$ in the two-dimensional $x-y$ plane, a total of six parameters. If one uses the Euclidean invariance of the $x-y$ plane to take out translations and rotations, one is left with three Euclidean-invariant initial conditions, for example the object-string separation distance $r$, the object speed

$$
v \equiv\left|\dot{\mathbf{r}}_{M}\right| \equiv \sqrt{\dot{\mathbf{r}}_{M} \cdot \dot{\mathbf{r}}_{M}},
$$

and the angle $\psi$ between the string-object separation vector $\mathbf{r}$ and the object velocity vector

$$
\mathbf{v} \equiv \dot{\mathbf{r}}_{M}
$$

defined so that

$$
\mathbf{r} \cdot \mathbf{v}=r v \cos \psi
$$

Instead of $\psi$, it is sometimes convenient below to use

$$
C \equiv \cos \psi=\frac{\mathbf{r} \cdot \mathbf{v}}{r v}
$$

or

$$
X \equiv \sin \frac{\psi}{2}=\sqrt{\frac{1-C}{2}}=\sqrt{\frac{r v-\mathbf{r} \cdot \mathbf{v}}{2 r v}} .
$$

Note that the $v$ given by Eq. (39) as the speed of the object in the frame in which the string was initially at rest, and used with this meaning henceforth in this paper, is not the same as the $v_{r}$ used in Section 2, e.g., in Eq. (16), which denoted $|\dot{\mathbf{r}}|$, the instantaneous relative speed of the object and the string, and it is also not the same as the $v_{s}$ used in Section 3, which by Eq. (19) denoted what we are now calling $\left|\dot{\mathbf{r}}_{s}\right|$, the speed of the point on the string nearest the object, in the frame in which the string was initially at rest.

If one takes out the time-translation invariance, one is left with only two initial conditions, which can be the initial speed $v_{0}$ at $t=-\infty$ and the impact parameter $b=r \sin \psi$ at $t=-\infty$, where $r$ is infinite and $\psi$ is $-\pi$. There Eqs. (38) imply that $\dot{\mathbf{r}}_{s}=0$, and one can choose the initial string position to be $\mathbf{r}_{s}=0$ (the string initially at rest at $x_{s}=y_{s}=0$ ) and the object to be coming in from $x_{M}=-\infty$ with $y_{M}=b$ and with velocity $v_{0}$ in the positive $x$-direction initially at $t=-\infty$. These initial conditions and the first of Eqs. (38) imply that

$$
\mathbf{r}_{s}=\mathbf{v}_{0}-\dot{\mathbf{r}}_{M}=\mathbf{v}_{0}-\mathbf{v}
$$

Inserting this into the second of Eqs. (38) gives a single second-order differential equation for the object vector position $\mathbf{r}_{M}(t)$, although the second term in the rightmost expression of Eqs. (38) makes this single equation nonlinear in $\ddot{\mathbf{r}}_{M}$.

As the object comes in from infinity, it will be pulled toward the string, so that at first it will speed up and bend downward, and $y_{M}$ will decrease below its initial 
value of $b$. Eventually the object will either be captured by the string (so that $r$ and $v$ tend to zero), or it scatter so that $r$ increases to infinity again, depending on the initial velocity $v_{0}$ and the impact parameter $b$. For each $v_{0}$, the boundary between these two qualitatively different asymptotic behaviors is given by the critical impact parameter $b_{\text {crit }}\left(v_{0}\right)$ : for $b<b_{\text {crit }}\left(v_{0}\right)$ the object is captured, and for $b>b_{\text {crit }}\left(v_{0}\right)$ the object scatters to $r=\infty$ with $v$ asymptotically greater than zero. For $b=b_{\text {crit }}\left(v_{0}\right)$, the marginal capture case, $r$ reaches a minimum and then slowly increases back to $\infty$, but with $v$ asymptotically decreasing toward zero, so that the object essentially has precisely the escape velocity from the string.

The main goal of this paper, besides deriving the coupled equations of motion (34) or (38) given above, is to calculate the critical impact parameter $b_{\text {crit }}\left(v_{0}\right)$ as a function of the initial velocity $v_{0}$ when this velocity is low compared with the speed of light. I shall derive one approximate formula when $L_{u} / T_{u} \ll v_{0} \ll c$, the regime in which the string acts essentially as a test string at the critical impact parameter (at least until $r$ reaches its minimum value; during the late stages when $r$ slowly increases back to $\infty$, the back reaction of the string on the object becomes important), and another approximate formula when $v_{0} \ll L_{u} / T_{u} \ll c$, the regime in which the string acts stiffly and is only slightly perturbed by the force from the object (though this small perturbation is essential for causing the object to lose enough energy to become bound).

In this paper I shall focus on the case in which the ratio of the second term to the first term on the extreme right hand side of Eqs. (38), $4 \mu r(\dot{\mathbf{r}} \cdot \dot{\mathbf{r}})$, is much less than unity, so we shall omit the last term of Eqs. (38). For the dominant part of the scattering, $r$ is of the order of magnitude of the impact parameter $b$, and $\dot{\mathbf{r}} \cdot \dot{\mathbf{r}}$ is of the order of magnitude of the initial velocity $v_{0}$, so this ratio is of the order of magnitude of $\mu b v_{0}^{2}$ (in units with $T_{u}=L_{u}=1$ ). For $v_{0} \ll L_{u} / T_{u}$, we shall find below that $b_{\text {crit }} \propto v_{0}^{-7 / 5}$, so for very small $v_{0}$, certainly $\mu b v_{0}^{2} \ll 1$ for the case of marginal capture. For $v_{0}$ of order unity $\left(L_{u} / T_{u}\right)$, the truncated Eqs. (38) (with the last term omitted) have no small or large parameters, so we expect that then $b_{\text {crit }}$ is also of order unity $\left(L_{u}\right)$, giving $\mu b_{\text {crit }} v_{0}^{2} \sim \mu \ll 1$. For $v_{0} \gg 1, b_{\text {crit }} \approx 2 v_{0}^{-1 / 2}$, so $\mu b_{\text {crit }} v_{0}^{2} \sim \mu v_{0}^{3 / 2}$. This is also small compared to unity when $v_{0} \ll \mu^{-2 / 3} \sim\left(1-Q^{2} / M^{2}\right)^{1 / 3} c$.

Therefore, for calculating the critical impact parameter $b_{\text {crit }}\left(v_{0}\right)$, it appears to be a good approximation to neglect the last term of Eqs. (38) for velocities low compared with the speed of light times the cube root of $\left(1-Q^{2} / M^{2}\right)$. Unless the object is significantly charged (e.g., nearly extremely charged if the object is a black hole), the limitation of considering only velocities low compared with the speed of light is sufficient for dropping the last term of Eqs. (38) when one calculates $b_{\text {crit }}\left(v_{0}\right)$. It is apparently not sufficient when $\left(1-Q^{2} / M^{2}\right)^{1 / 3} c \ll v_{0} \ll c$, but the calculation of $b_{\text {crit }}\left(v_{0}\right)$ in this regime for nearly extremely charged objects will be left as an exercise for the reader.

When we drop the last term of Eqs. (38), use Eq. (44) to solve the first part of 
these equations, and define

$$
\mathbf{R}=\mathbf{r}_{M}-\mathbf{v}_{0},
$$

then what remains of Eqs. (38) becomes the single second-order vector equation

$$
\ddot{\mathbf{R}}=-\frac{\mathbf{R}+\dot{\mathbf{R}}}{|\mathbf{R}+\dot{\mathbf{R}}|^{3}} .
$$

In terms of $\mathbf{R}$, one has

$$
\begin{gathered}
\mathbf{r}_{M}=\mathbf{v}_{0}+\mathbf{R}, \\
\mathbf{r}_{s}=\mathbf{v}_{0}-\dot{\mathbf{R}}, \\
\mathbf{r}=\mathbf{R}+\dot{\mathbf{R}}, \\
\mathbf{v} \equiv \dot{\mathbf{r}}_{M}=\dot{\mathbf{R}} .
\end{gathered}
$$

Alternatively, Eq. (46) can be written as two first-order vector equations,

$$
\begin{gathered}
\dot{\mathbf{r}}=\mathbf{v}-\frac{\mathbf{r}}{r^{3}}, \\
\dot{\mathbf{v}}=-\frac{\mathbf{r}}{r^{3}} .
\end{gathered}
$$

If we instead go to the three Euclidean-invariant quantities $r, v$, and either $C=$ $\cos \psi=\mathbf{r} \cdot \mathbf{v} /(r v)$ or $X=\sin (\psi / 2)=\sqrt{(1-C) / 2}$, one gets the three first-order scalar equations

$$
\begin{gathered}
\dot{r}=C v-\frac{1}{r^{2}}, \\
\dot{v}=-\frac{C}{r^{2}},
\end{gathered}
$$

and either

or

$$
\dot{C}=\left(1-C^{2}\right)\left(\frac{v}{r}-\frac{1}{r^{2} v}\right)
$$

$$
\dot{X}=-X\left(1-X^{2}\right)\left(\frac{v}{r}-\frac{1}{r^{2} v}\right) .
$$

Another choice of variables is motivated by the fact that in the stiff-string regime, where $r^{2} v \gg 1$, the equations are approximately those of a unit-mass Keplerian particle orbiting a stationary string that exerts a unit inverse-square force on the particle, $\dot{\mathbf{r}} \approx \mathbf{v}$ and $\dot{\mathbf{v}}=-\mathbf{r} / r^{3}$. For such a particle, conserved quantities are the energy,

$$
E \equiv \frac{1}{2} v^{2}-\frac{1}{r}
$$

the angular momentum,

$$
L \equiv r v \sin \psi=r v \sqrt{1-C^{2}}=2 r v X \sqrt{1-X^{2}}
$$


one-eighth the cube of the angular momentum,

$$
h \equiv \frac{1}{8} L^{3}=r^{3} v^{3}\left(X^{2}-X^{4}\right)^{3 / 2},
$$

the eccentricity of the orbit,

$$
e \equiv \sqrt{1+2 E L^{2}}
$$

and one-fourth the excess of the square of the eccentricity over unity,

$$
g \equiv \frac{1}{4}\left(e^{2}-1\right)=\frac{1}{2} E L^{2}=\left(r^{2} v^{4}-2 r v^{2}\right) X^{2}\left(1-X^{2}\right)=\left[\left(r v^{2}-1\right)^{2}-1\right]\left(X^{2}-X^{4}\right) .
$$

Only two of these quantities are independent, and to get fairly simple differential equations below, I shall focus on $g$ and $h$. In terms of them and $X^{2}-X^{4}=$ $(1 / 4) \sin ^{2} \psi$, one may readily solve algebraically for $r$ and $v$ :

$$
\begin{gathered}
r=\frac{h^{2 / 3}}{\sqrt{X^{2}-X^{4}}\left(\sqrt{X^{2}-X^{4}}+\sqrt{X^{2}-X^{4}+g}\right)}, \\
v=h^{-1 / 3}\left(\sqrt{X^{2}-X^{4}}+\sqrt{X^{2}-X^{4}+g}\right) .
\end{gathered}
$$

Because the string is in actuality not completely stiff but moves toward the object, the quantities $E, L, h, e$, and $g$ are not precisely conserved in the coupled object-string equations but instead are all decreasing with time, at the rates

$$
\begin{gathered}
\dot{E}=-\frac{1}{r^{4}}, \\
\dot{L}=-\frac{L}{r^{3}}, \\
\dot{h}=-\frac{3 h}{r^{3}}=-3 h^{-1}\left(X^{2}-X^{4}\right)^{3 / 2}\left(\sqrt{X^{2}-X^{4}}+\sqrt{X^{2}-X^{4}+g}\right)^{3}, \\
\dot{e}=-\frac{L^{2}}{e r^{4}}\left(r v^{2}-1\right)=-\frac{v^{3}}{e} \dot{C}, \\
\dot{g}=-\frac{L^{2}}{2 r^{4}}\left(r v^{2}-1\right)=-\frac{1}{2} v^{3} \dot{C}=2 v^{3} X \dot{X} \\
-2 h^{-2}\left(X^{2}-X^{4}\right)^{3 / 2} \sqrt{X^{2}-X^{4}+g}\left(\sqrt{X^{2}-X^{4}}+\sqrt{X^{2}-X^{4}+g}\right)^{4} .
\end{gathered}
$$

Now we can insert Eq. (62) and Eq. (63) into Eq. (56) to get

$$
\dot{X}=-\frac{\left(X^{2}-X^{4}\right)^{3 / 2}}{h X} \sqrt{X^{2}-X^{4}+g}\left(\sqrt{X^{2}-X^{4}}+\sqrt{X^{2}-X^{4}+g}\right) .
$$


Then dividing $\dot{g}$ and $\dot{h}$ by $\dot{X}$ gives two coupled first-order differential equations for the coupled motion of the object and the string:

$$
\begin{aligned}
& \frac{d g}{d X}=\frac{2}{h} X\left(\sqrt{X^{2}-X^{4}}+\sqrt{X^{2}-X^{4}+g}\right)^{3} \\
& \frac{d h}{d X}=\frac{3 X\left(\sqrt{X^{2}-X^{4}}+\sqrt{X^{2}-X^{4}+g}\right)^{2}}{\sqrt{X^{2}-X^{4}+g}} .
\end{aligned}
$$

At $t=-\infty$, one has the object approaching the string, and so the angle $\psi$ between the object velocity $\mathbf{v}$ and the infinitely-long string-object separation vector $\mathbf{r}$ is $\pi$, giving $X=\sin (\psi / 2)=1$ initially. The energy is then $E_{0}=v_{0}^{2} / 2$, and the angular momentum is then $L_{0}=b v_{0}$, so

$$
\begin{gathered}
g(X=1)=\frac{1}{2} E_{0} L_{0}^{2}=\frac{1}{4} b^{2} v_{0}^{4}, \\
h(X=1)=\frac{1}{8} L_{0}^{3}=\frac{1}{8} b^{3} v_{0}^{3} .
\end{gathered}
$$

Then as $X$ decreases, so do $g$ and $h$. If the object scatters and remains unbound, the energy and hence $g$ remain positive as the object returns to infinitely large $r$, but now with the object moving away from the string, so $\mathbf{v}$ and $\mathbf{r}$ asymptotically approach the same direction, giving $\psi=0$ and hence $X=0$. If the object becomes bound to the string, $E=v^{2} / 2-1 / r$ becomes negative, and hence also $g$. Eq. (71) becomes singular where $\sqrt{X^{2}-X^{4}+g}$ goes to zero, but one can switch back to Eqs. (53)-(55) or (56) to continue the calculation, as $\dot{X}$ and $\sqrt{X^{2}-X^{4}+g}$ reverse sign and the evolution continues, with $X$ oscillating as the object spirals in to join the string after a finite time.

In the intermediate case in which the impact parameter $b$ has the critical value $b_{\text {crit }}\left(v_{0}\right), E$ and $g$ tend to zero as the object slowly moves to infinite separation $r$ with essentially just the escape velocity. In this case, like the scattering case in which $g$ stays positive, the object velocity $\mathbf{v}$ and the string-object separation vector $\mathbf{r}$ asymptotically become parallel, giving $X=0$ at $t=\infty$.

It is now easy to calculate a one-parameter family of $v_{0}$ 's and their corresponding $b_{\text {crit }}\left(v_{0}\right)$ 's using Eqs. (70) and (71), without requiring a shooting method from initial conditions at $t=-\infty$ or $X=1$. Instead, one evolves backward in time from $t=+\infty$ or $X=0$, where one sets $g(X=0)=0$ but uses

$$
\eta \equiv h(X=0)=\frac{1}{8} L^{3}(t=\infty)
$$

as the single free parameter for determining the one-parameter family of critical solutions. Each solution is evolved to $X=1$, where one evaluates $g(\eta, X=1)$ and $h(\eta, X=1)$ and then inverts Eqs. (72) and (73) to get

$$
\begin{gathered}
v_{0}(\eta)=g(\eta, X=1)^{1 / 2} h(\eta, X=1)^{-1 / 3}, \\
b_{\text {crit }}\left(v_{0}(\eta)\right)=2 g(\eta, X=1)^{-1 / 2} h(\eta, X=1)^{2 / 3} .
\end{gathered}
$$




\section{The critical impact parameter in the stiff-string regime, $v_{0} \ll L_{u} / T_{u}$}

Now we shall find approximate analytic solutions of the evolution Eqs. (70) and (71) in the marginally bound case $(g(X=0)=0$ or $E(t=\infty)=0)$ in the two limiting cases in which $\eta=h(X=0)=L^{3}(t=\infty) / 8$ is either very large or very small.

For $\eta \gg 1$, the factor of $h$ in the denominator of Eq. (70) means that to lowest order $g(X)$ stays very small and can be neglected on the right hand sides of Eqs. (70) and (71). Then one gets

$$
\begin{aligned}
g(X) & \approx \frac{1}{64 \eta}(12 \psi-8 \sin 2 \psi+\sin 4 \psi) \\
& =\frac{1}{8 \eta}\left[3 \sin ^{-1} X+\left(-3-2 X^{2}+24 X^{4}-16 X^{6}\right) \sqrt{X^{2}-X^{4}}\right], \\
h(X) \approx \eta+ & \frac{3}{8}(2 \psi-\sin 2 \psi)=\eta+\frac{3}{2}\left[\sin ^{-1} X+\left(-1+2 X^{2}\right) \sqrt{X^{2}-X^{4}}\right] .
\end{aligned}
$$

This is sufficiently accurate to give

$$
h(\eta, X=1)=\eta\left[1+\frac{3 \pi}{4 \eta}+O\left(\frac{1}{\eta^{2}}\right)\right] .
$$

However, to get $g(\eta, X=1)$ with a relative error of only $O\left(\eta^{-2}\right)$ rather than $O\left(\eta^{-1}\right)$, one needs to insert the approximate expressions (77) and (78) into the right hand side of Eq. (70) and evaluate it through the next-to-lowest order in $\eta^{-1}$. This gives

$$
g(\eta, X=1)=\frac{3 \pi}{16 \eta}\left[1+\frac{0}{\eta}+O\left(\frac{1}{\eta^{2}}\right)\right]
$$

If we insert these expressions into Eqs. (75) and (76) to get $v_{0}(\eta)$ and $b_{\text {crit }}(\eta)$ and then eliminate $\eta$ to get $b_{\text {crit }}\left(v_{0}\right)$, we find that

$$
b_{\text {crit }}=(6 \pi)^{1 / 5} v_{0}^{-7 / 5}+\frac{1}{5}(6 \pi)^{3 / 5} v_{0}^{-1 / 5}+O\left(v_{0}\right) .
$$

Large $\eta$ gives small $v_{0} \approx \sqrt{3 \pi} /\left(4 \eta^{5 / 6}\right)$, so Eq. (81) applies for $v_{0} \ll L_{u} / T_{u} \equiv 1$. At the critical impact parameter, this is the stiff-string regime, where the string responds much less than the object to the force between them. If one restores the units $T_{u}$ and $L_{u}$ given by Eqs. (35) and (36), one obtains the formula given in the Abstract above for the stiff-string regime.

One can give an order-of-magnitude derivation of the critical impact parameter to show that it indeed goes as $v_{0}^{-7 / 5}$ for $v_{0} \ll 1$. What happens is that the object comes in on a slightly hyperbolic, but nearly parabolic, orbit with initial energy $E_{0}=v_{0}^{2} / 2$ 
just slightly positive. Then as the object swings around the string, the small motion of the string toward the object drains just enough energy from the object that it goes back out on an asymptotically parabolic orbit with $E$ approaching zero.

In this analysis, use $\approx$ for approximate numerical equality under the limits given (i.e., $x \ll 1$ implies that $1+x \approx 1$ ), and use $\sim$ for order-of-magnitude equality that has the correct powers of small quantities (e.g., $2 x \sim x$ ).

With impact parameter $b \gg 1 / v_{0}$, the angular momentum $L$ stays nearly constant at its initial value $L_{0}=b v_{0} \gg 1$ (as I shall show momentarily), so at the perispaggon, the point on the object orbit nearest the string, the minimal separation $r_{m}$ and the object speed $v_{m}$ are related by $r_{m} v_{m} \approx L \approx L_{0}=b v_{0}$. Assuming that $E=v^{2} / 2-1 / r \ll 1 / b$ (which for $E \sim E_{0}$ requires $b \ll 1 / v_{0}^{2}$, so we need $1 / v_{0} \ll b \ll 1 / v_{0}^{2}$ and hence $v_{0} \ll 1$ ), we get $v_{m}^{2} / 2 \approx 1 / r_{m} \gg v_{0}^{2} / 2$. Combining this with $r_{m} v_{m} \approx L_{0}$ then gives

$$
\begin{gathered}
r_{m} \approx \frac{1}{2} L_{0}^{2}, \\
v_{m} \approx \frac{2}{L_{0}} .
\end{gathered}
$$

The time spent near perispaggon is $t_{m} \sim r_{m} / v_{m} \sim L_{0}^{3}$. During this time, the angular momentum decreases at the rate given by Eqs. (65), $\dot{L}=-L / r^{3} \sim-L_{0} / r_{m}^{3} \sim$ $-1 / L_{0}^{5}$, and thus it decreases by a total amount roughly $t_{m}$ as large, or $\Delta L \sim-1 / L_{0}^{2}$. The magnitude of this decrease is much less than $L_{0}$ for $L_{0} \gg 1$ or $b \gg 1 / v_{0}$, as claimed above, so in this regime the angular momentum is approximately constant.

The energy decreases at the rate given by Eqs. (74), $\dot{E}=-1 / r^{4} \sim-1 / r_{m}^{4} \sim$ $1 / L_{0}^{8}$, and multiplying this by the time $t_{m}$ gives a total decrease $-\Delta E \sim 1 / L_{0}^{5} \approx$ $1 /\left(b v_{0}\right)^{5}$. For the final energy to be zero, this decrease needs to equal the initial energy, giving $v_{0}^{2} \sim E_{0} \sim-\Delta E \sim 1 /\left(b v_{0}\right)^{5}$ or $b=b_{\text {crit }} \sim v_{0}^{-7 / 5}$. For $v_{0} \ll 1$, this is indeed within the allowed range $1 / v_{0} \ll b_{\text {crit }} \sim v_{0}^{-7 / 5} \ll 1 / v_{0}^{2}$ of the approximations used above.

One can easily get the correct coefficient $(6 \pi)^{1 / 5}$ of the $v_{0}^{-7 / 5}$ term for $b_{\text {crit }}$ by calculating the time integral of the energy loss rate $\dot{E}=-1 / r^{4}$ for a parabolic orbit of angular momentum $L_{0}=b v_{0}$, but that confirmation of the precise form of the first term of the right hand side of Eq. (81) will be left as an exercise for the reader. Similarly, one should be able to get the second term on the right hand side of Eq. (81) from first-order perturbations due to the motion of the string and the resultant loss of the energy and angular momentum of the object.

\section{The critical impact parameter in the test-string regime, $v_{0} \gg L_{u} / T_{u}$}

Now I shall consider the opposite case, $\eta \equiv h(X=0)=L^{3}(t=\infty) / 8 \ll 1$, which leads to $v_{0} \gg L_{u} / T_{u} \equiv 1$ and $b_{\text {crit }} \ll L_{u} \equiv 1$. This is the test-string limit, for 
which the lowest-order approximation is to ignore the string tension and the effect of the string on the object. The critical impact parameter in this limit was found in [3] to be $b_{\text {crit }} \simeq 2 v_{0}^{-1 / 2}$ after converting to the units used in this paper. Here I shall rederive this result and give the lowest-order correction in the small quantity $1 / v_{0}$.

In this test-string regime $v_{0} \gg 1$, the qualitative picture is that initially the object moves with very nearly constant velocity, and the string gets bent toward the object as described in [3]. In that paper, where the back reaction of the string on the object was totally ignored, the final situation, when the impact parameter $b$ has the critical value $b_{\text {crit }}$ for marginal capture, is for the string to develop a kink that asymptotically trails the object at a distance of $v_{0}^{-1 / 2}=b_{\text {crit }} / 2$ behind it, an unstable equilibrium point where the force of the object on the string causes the string to follow the object at precisely the same speed as the object, by Eq. (32). In the complete test-string approximation, the deceleration of the object is ignored, so it continues moving forever, pulling an ever longer kink of string behind it.

When one does include the back reaction effect of the string tension on the object but stays in the test-string regime of $v_{0} \gg 1$, the picture during the first stage of the evolution in the marginal capture case is very similar to that of the complete test-string approximation used in [3], so the position on the string nearest the object approaches quite near to what would be the unstable equilibrium separation behind the object if the object continued moving with constant velocity. But then in actuality the object slows down as the string weakly pulls back on it (somewhat analogous to the way a fish slows down when attached to a weak fishing line [12]). If it had been at the unstable equilibrium separation for the velocity of the object (i.e., the separation that would be the equilibrium one if the object continued moving with constant velocity), then the slowing down of the object would eventually lead to its falling into the string. For the object to be just marginally not falling into the string, the separation must be greater, by a precise tiny amount, than what would be the equilibrium separation at constant velocity. At this slightly greater separation, the force on the kink of the string trailing the object would be just slightly less than needed to move it at the speed of the object, so the string moves slightly slower than the object and hence trails behind it at an indefinitely increasing separation, gradually slowing down more and more.

If the object had had an impact parameter $b$ slightly greater than $b_{\text {crit }}\left(v_{0}\right)$, it would maintain a velocity bounded away from zero as its separation from the string increased indefinitely. On the other hand, if the object had $b$ slightly smaller than $b_{\text {crit }}\left(v_{0}\right)$, the object would eventually slow down more than the string, so that the separation would stop increasing and would henceforth decrease to zero as the object falls into the string. But in the marginal capture case with impact parameter $b=$ $b_{\text {crit }}\left(v_{0}\right)$, the separation $r$ between the string and the object, although continuing to grow indefinitely, grows slowly enough that the object, as well as the string, continues to slow down just fast enough that it asymptotically approaches, but never reaches, 
zero velocity.

We can put this discussion into a more quantitative form in the following way: For $g \ll X^{2} \ll 1$, Eqs. (70) and (71) become

$$
\begin{gathered}
\frac{d g}{d X}=\frac{16 X^{4}+12 X^{2} g+O\left(X^{6}\right)+O\left(g^{2}\right)}{h} \\
\frac{d h}{d X}=12 X^{2}+O\left(X^{4}\right)+O\left(g^{2}\right)
\end{gathered}
$$

with the critical solutions $(g=0$ and $h=\eta>0$ at $X=0)$

$$
\begin{gathered}
g(X)=\frac{16}{5}\left(\frac{X^{5}}{\eta}-\frac{X^{8}}{\eta^{2}}\right)+O\left(\frac{X^{7}}{\eta}\right)+O\left(\frac{X^{10}}{\eta^{2}}\right)+O\left(\frac{X^{11}}{\eta^{3}}\right) \\
h(X)=\eta+4 X^{3}+O\left(X^{5}\right)+O\left(\eta^{-1}\right) .
\end{gathered}
$$

To get the time dependence of these solutions, we can use Eq. (69), which for $g \ll X^{2} \ll 1$ becomes

$$
\dot{X}=\frac{4 X^{4}+3 X^{2} g+O\left(X^{6}\right)+O\left(g^{2}\right)}{2 h} .
$$

Inserting $g(X)$ and $h(X)$ from Eqs. (86) and (87) into this and integrating gives

$$
t=\frac{\eta}{6 X^{3}}-\frac{4}{5} \ln X+O\left(\frac{X^{3}}{\eta}\right)
$$

dropping the irrelevant constant of integration. For large $t$ this can be inverted to give

$$
X=\left(\frac{\eta}{6 t}\right)^{1 / 3}\left[1+\frac{4}{45 t} \ln \frac{6 t}{\eta}+O\left(\frac{\ln ^{2} t}{t^{2}}\right)\right],
$$

Then from Eqs. (86), (87), (62), and (63), one may get

$$
\begin{gathered}
g(t) \approx \frac{16}{5} \eta^{2 / 3}(6 t)^{-5 / 3}\left(1+\frac{4}{9 t} \ln \frac{6 t}{\eta}\right), \\
h(t) \approx \eta\left(1+\frac{3}{2 t}+\frac{8}{45 t^{2}} \ln \frac{6 t}{\eta}\right), \\
r(t) \approx \frac{1}{2}(6 t)^{2 / 3}\left(1-\frac{8}{45 t} \ln \frac{6 t}{\eta}\right), \\
v(t) \approx 2(6 t)^{-1 / 3}\left(1+\frac{4}{45 t} \ln \frac{6 t}{\eta}\right) .
\end{gathered}
$$

Alternatively, one can divide Eq. (53) by Eq. (54) to get

$$
\frac{d r}{d v}=\sec \psi-r^{2} v \approx 1-r^{2} v
$$


for $X \equiv \sin \psi / 2 \ll 1$, where one may recall that $\psi$ is the angle between the velocity of the object and the string-object separation, so $\psi$ goes from $\pi$ initially (as the object heads toward the string) to 0 finally for marginal capture (as the object heads away from the string with slower and slower velocity). When $X$ can be neglected, Eq. (95) leads to the series solution

$$
\frac{1}{r} \approx \frac{1}{2} v^{2}-\frac{1}{20} v^{5}+\frac{1}{160} v^{8}-\frac{7}{8800} v^{11}+\frac{1}{9856} v^{14}+O\left(v^{17}\right)
$$

or

$$
v(r) \approx \sqrt{2} r^{-1 / 2}+\frac{1}{5} r^{-2}+\frac{\sqrt{2}}{25} r^{-7 / 2}+\frac{24}{1375} r^{-5}+O\left(r^{-13 / 2}\right) .
$$

All of the series expansions given so far in this section are good only when $t \gg 1$, $r \gg 1$, and $v \ll 1$, in addition to $X \ll 1$ (i.e., when the object is moving very slowly nearly directly away from the string at very late times). When $\eta \ll 1$, as one integrates backward in time from the final condition $g=0$ and $h=\eta$ at $X=0$ (where $t=\infty, r=\infty$, and $v=0$ ), the approximate second equality of Eq. (95) remains valid so long as $X \ll 1$ and gives, when $v$ becomes very large and $r$ becomes very small,

$$
\frac{1}{r} \approx v^{1 / 2}-\frac{1}{4} v^{-1}-\frac{5}{32} v^{-5 / 2}-\frac{15}{64} v^{-4}-\frac{1105}{2048} v^{-11 / 2}+O\left(v^{-7}\right),
$$

up to terms that decrease roughly as the exponential of $-(4 / 3) v^{3 / 2}$, so long as $X$ remains negligible. Inverting this gives

$$
v(r) \approx \frac{1}{r^{2}}+\frac{1}{2} r+\frac{1}{8} r^{4}+\frac{5}{32} r^{7}+O\left(r^{10}\right)
$$

for small $r$, again neglecting all contributions from nonzero $X$ and terms exponential in $-4 /\left(3 r^{3}\right)$.

To get the $X$-dependence of the critical solution when $X \ll 1$ and to prepare the way for the corrections when $X$ is no longer small, divide Eq. (56) by Eq. (54) to get

$$
\frac{d X}{d v}=\frac{X\left(1-X^{2}\right)}{1-2 X^{2}}\left(1-\frac{1}{r v^{2}}\right) r v \approx X\left(1-\frac{1}{r v^{2}}\right) r v,
$$

with the approximate equality applying when $X \ll 1$.

When $v \ll 1$ so that Eq. (96) is applicable, Eq. (100) becomes

$$
\frac{d X}{d v} \approx \frac{X}{v},
$$

which, when matched to the boundary condition that $L=2 r v X \sqrt{1-X^{2}}$ is equal to $2 \eta^{1 / 3}$ at $X=0$, gives

$$
X \approx \frac{1}{2} \eta^{1 / 3} v \approx \frac{\eta^{1 / 3}}{\sqrt{2 r}}
$$


for $v \ll 1, r \gg 1$, or $X \ll \eta^{1 / 3}$.

At $X \sim \eta^{1 / 3} \ll 1, v$ surpasses unity and thereafter becomes large, and $r$ becomes small (until $X$ gets near 1 and $r$ becomes large again in this evolution backward in time). When $v \gg 1$ so that Eq. (98) is applicable, with $r v^{2} \approx v^{3 / 2} \gg 1$, Eq. (100) becomes

$$
\frac{d X}{d v} \approx \frac{X\left(1-X^{2}\right) r v}{1-2 X^{2}} \approx X r v
$$

where the first approximate equality applies for all $X \gg \eta^{1 / 3}$ and the second approximate equality applies for $\eta^{1 / 3} \ll X \ll 1$.

Eq. (103), along with Eq. (98), implies that once $v$ gets large, $X$ grows roughly exponentially with $(2 / 3) v^{3 / 2}$. After $X$ passes $v^{-1 / 2}, d v / d X$ becomes small, and the large $v$ changes very little during the rest of the backward evolution to the initial conditions at $X=0\left(t=-\infty, v=v_{0}\right)$. Since this exponential growth of $X$ with $v$ starts at $X \sim \eta^{1 / 3}$ where $v \sim 1$ and ends at $X \sim 1$ where $v \sim v_{0}$, one gets

$$
v_{0} \sim\left(-\frac{1}{2} \ln \eta\right)^{2 / 3}
$$

for very small $\eta$. This very slow growth of $v_{0}$ with $1 / \eta$ implies that when one solves Eqs. (70) and (71) numerically with the numerical initial conditions $g(X=0)=0$ and $h(X=0)=\eta$ (final conditions in time for marginal capture), one must choose $\eta$ to be extremely tiny to get a large value of $v_{0}$, and for the approximations of this section, one must assume by $\eta \ll 1$ not just that $\eta$ is more than an order of magnitude (say) smaller than unity, but that $-\ln \eta$ is more than an order of magnitude (say) larger than unity.

Once the marginal capture solution for $\eta \ll 1$ has been carried past $X \sim$ $(-\ln \eta)^{-1 / 3}$ where $d v / d X$ becomes small, $v$ stays nearly constant and one is in the test-string regime treated in [3], where one can to first approximation neglect the back reaction of the string on the object (the object's acceleration toward the string). In this paper, however, we want to treat this back reaction perturbatively in this test-string regime and get the first-order correction to the test-string critical impact parameter formula $b_{\text {crit }} \simeq 2 v_{0}^{-1 / 2}$ given in [3].

For this purpose it helps to change the dependent variables of integration from the $g(X)$ and $h(X)$ used in Eqs. (70) and (71) to two other variables that would be constant if the test-string approximation were completely accurate. One such variable is $v$, the magnitude of the velocity of the object (in the frame in which the string was initially at rest along the $z$-axis). A second such variable is motivated by the test-string Eq. (67) of [3], where the polar coordinate angle $\theta$ defined just before Eq. (54) of that paper is essentially the same as $\psi$ here, where $r$ there is the same as $r$ here, where $z$ given there just before Eq. (54) as $r \sin \theta$ is hence the same as $r \sin \psi=2 r X \sqrt{1-X^{2}}$ in the notation of the present paper, and where the constant $L$ given by Eq. (50) (not to be confused with the angular momentum $L$ 
of the present paper) is the same as $v_{0}^{-1 / 2}$ in our present units $T_{u} \equiv 1$ and $L_{u} \equiv 1$. Then Eq. (67) of [3], plus the equation $b_{\text {crit }} \simeq 2 L$ three lines below, imply that

$$
b^{2}-b_{\mathrm{crit}}^{2} \simeq 4 r^{2} X^{2}\left(1-X^{2}\right)-4 v_{0}^{-1} X^{2}
$$

is a constant of motion in the test-string approximation, and furthermore that it is zero for marginal capture. If we use the fact that $v \simeq v_{0}$ to replace $v_{0}$ by $v$ and then multiply the expression on the right hand side by $v / 4$, we get the quantity

$$
w \equiv r^{2} v\left(X^{2}-X^{4}\right)-X^{2}=\frac{L^{2}}{4 v}-X^{2}=\frac{h}{\sqrt{X^{2}-X^{4}}+\sqrt{X^{2}-X^{4}+g}}-X^{2} .
$$

Eqs. (70) and (71) now imply that the alternative dependent variables $v$ and $w$ obey the differential equations

$$
\begin{aligned}
& \frac{d v}{d X}=\frac{X\left(1-2 X^{2}\right)}{\sqrt{v\left(X^{2}-X^{4}\right)\left(X^{2}+w\right)}}\left[1-\sqrt{\frac{X^{2}-X^{4}}{v^{3}\left(X^{2}+w\right)}}\right]^{-1}, \\
& \frac{d w}{d X}=\frac{X\left[X^{2}-\left(1-2 X^{2}\right) w\right]}{\sqrt{v^{3}\left(X^{2}-X^{4}\right)\left(X^{2}+w\right)}}\left[1-\sqrt{\frac{X^{2}-X^{4}}{v^{3}\left(X^{2}+w\right)}}\right]^{-1} .
\end{aligned}
$$

We can see from Eq. (106) that $w$ is not a convenient variable to integrate all the way from $X=0$, since sufficiently near there that $h \approx \eta$ and $g \ll 1$, the last expression of Eqs. (106) implies that we have $w \approx h /(2 X)$, which diverges at $X=0$. Alternatively, in the second-to-last expression of Eq. (106), one gets a divergence from the fact that $L$ goes to the nonzero constant $2 \eta^{1 / 3}$ at $X=0$, but $v$ goes to zero there. However, from Eq. (98) for $\eta^{1 / 3} \ll X \ll 1$, where $v \gg 1$ and $r \approx v^{-1 / 2} \ll 1$, one can see that then $w \approx(1 / 2) v^{-3 / 2} X^{2}$, which is negligibly small for small enough $X$. E.g., for $X \sim \eta^{1 / 3} \sim e^{-(2 / 3) v_{0}^{3 / 2}}$, where $v \sim 1$ and $r \sim 1, w \sim e^{-(2 / 3) v_{0}^{3 / 2}}$ is exponentially small for large $v_{0}$. Therefore, with only an exponentially small error, we can set $w=0$ and $v \gg 1$ as initial conditions in integrating Eqs. (107) and (108) from some very small $X$, such as $\eta^{1 / 3}$, which for practical purposes we can replace with 0 with only an additional exponentially small error.

Because Eqs. (107) and (108) have $v^{1 / 2} \gg 1$ and $v^{3 / 2} \gg 1$, respectively, in the denominators of the right hand sides, whereas the other factors are of order unity (except for harmless square-root singularities in $1-X^{2}$ at $X=1$ ), $v$ and $w$ change by only small amounts as one integrates to $X=1$ to get the temporally initial conditions for marginal capture. To lowest order in $1 / v_{0}$, one can thus neglect $w$ on the right hand sides and rewrite Eqs. (107) and (108) as

$$
\frac{d v}{d X} \approx \frac{1-2 X^{2}}{\sqrt{v\left(X^{2}-X^{4}\right)}} \approx \frac{1-2 X^{2}}{\sqrt{v_{0}\left(X^{2}-X^{4}\right)}}
$$




$$
\frac{d w}{d X} \approx \frac{X^{2}}{\sqrt{v^{3}\left(X^{2}-X^{4}\right)}} \approx \frac{X^{2}}{\sqrt{v_{0}^{3}\left(X^{2}-X^{4}\right)}} .
$$

Then Eq. (110) integrates to give

$$
w(X=1)=\frac{L_{0}}{4 v_{0}}-1=\frac{1}{4} b_{\text {crit }}^{2} v_{0}-1=v_{0}^{-3 / 2}+O\left(v_{0}^{-3}\right) .
$$

From this we can readily solve for the critical impact parameter, in the units of $T_{u}$ and of $L_{u}$ given by Eqs. (35) and (36), for large $v_{0} \gg L_{u} / T_{u} \equiv 1$ :

$$
b_{\text {crit }}\left(v_{0}\right)=2 v_{0}^{-1 / 2}+v_{0}^{-2}+O\left(v_{0}^{-7 / 2}\right) .
$$

When the units are restored, one gets the result given in the Abstract.

An order-of-magnitude argument for the $v_{0}^{-1 / 2}$ power law of the leading term was given in [3]. Summarizing it very briefly in the units used here, if the object is incident with velocity $v_{0} \gg 1$ and impact parameter $b$ such that it is not captured, the distance of closest approach will be $r \sim b$, the velocity at that point will be $v \sim v_{0}$, the time when the string is roughly that close will be $\sim r / v \sim b / v_{0}$, the length of string that bends significantly will be the speed of light times this or $\sim c b / v_{0}$, the bending angle will be $\sim 1 /\left(c r^{2}\right) \sim 1 /\left(c b^{2}\right)$, and hence the total transverse bending of the string will be the length of string significantly bent times this bending angle, or $\sim 1 /\left(b v_{0}\right)$. Marginal capture requires that this total transverse bending be of the same order as the impact parameter, giving $b=b_{\text {crit }} \sim 1 / \sqrt{v_{0}}$.

\section{The critical impact parameter over the entire range of nonrelativistic velocities}

Now that we have found analytically Eq. (81) for the critical impact parameter at very low initial velocities, $v_{0} \ll L_{u} / T_{u} \ll c$, and Eq. (112) for $b_{\text {crit }}\left(v_{0}\right)$ at high (but still nonrelativistic) velocities, $L_{u} / T_{u} \ll v_{0} \ll c$, we would like to find it numerically for all nonrelativistic velocities, and ideally find a fairly simple formula that gives a good fit to $b_{\text {crit }}\left(v_{0}\right)$ for all $v_{0}$.

It was straightforward to program Mathematica 2.0 on my ancient NeXT to integrate Eqs. (70) and (71) numerically from the temporally final conditions $g=0$ and $h=\eta$ at $X=0$ back to the temporally initial conditions at $X=0$ and then use Eqs. (75) and (76) to solve for $v_{0}$ and $b_{\text {crit }}\left(v_{0}\right)$. It is slightly awkward that by this method one cannot straightforwardly choose the values of $v_{0}$ for which one gets $b_{\text {crit }}\left(v_{0}\right)$, but this is a small price to pay for not having to use a shooting method to find the critical or marginally bound solutions. (One could use a shooting method to get any particular $v_{0}$ desired, but for determining the characteristics of the function $b_{\text {crit }}\left(v_{0}\right)$, this is hardly necessary.) 
Another slight awkwardness is that to get large values of the initial velocity $v_{0}$, one must use exponentially small values of $\eta$, and this can lead to some numerical inaccuracy, but this was not a serious problem for at least moderately large values of $v_{0}$.

I calculated over 50 pairs of $\left(v_{0}, b_{\text {crit }}\left(v_{0}\right)\right)$, ranging from $(0.00104198,26910)$ through (for example) $(0.834385,3.48671)$ to $(357.89,0.105727)$. Larger values of $v_{0}$ require such extraordinarily small values of $\eta$ that it would be somewhat difficult by my numerical method to obtain them, but what I could easily attain extends well into the domain of validity of both Eq. (81) and Eq. (112).

The results from the numerical integrations fit extremely well to the truncated series expansions of Eq. (81) at very small $v_{0}$ and of Eq. (112) at large $v_{0}$, confirming both the two coefficients and the two exponents of each of the two expressions (eight parameters calculated analytically and confirmed numerically).

One can combine Eqs. (81) and (112) into the interpolation formula

$$
b_{\text {crit }}\left(v_{0}\right) \simeq B_{(\alpha, \beta)}\left(v_{0}\right) \equiv\left[\left(\frac{6 \pi}{v_{0}^{7}}\right)^{\frac{\alpha}{5}}+\left(\frac{4}{v_{0}}\right)^{\frac{\alpha}{2}}\right]^{\frac{1}{\alpha}}+\left[\left(\frac{5^{5} v_{0}}{6^{3} \pi^{3}}\right)^{\frac{\beta}{5}}+v_{0}^{2 \beta}\right]^{-\frac{1}{\beta}},
$$

which depends not only on the eight parameters (four coefficients and four exponents) calculated analytically and given in Eqs. (81) and (112), but also on two new parameters, $\alpha$ and $\beta$. Let us see how well this formula works for suitable $\alpha$ and $\beta$.

In order that the first two terms of the right hand side of Eq. (81) be the dominant terms of the right hand side of Eqs. (113) at $v_{0} \ll 1$, one needs $\alpha>4 / 3$ and $\beta>0$, and in order that the first two terms of the right hand side of Eq. (112) be the dominant terms of the right hand side of Eqs. (113) at $v_{0} \gg 1$, one needs $\alpha>5 / 3$ and $\beta>0$. The combination of these two pairs of inequalities gives what might be called Criterion $\mathrm{A}, \alpha>5 / 3$ and $\beta>0$. If one wants to avoid any deviations larger than the $O\left(v_{0}\right)$ term of Eq. (81) at small $V_{0}$, one needs $\alpha \geq 8 / 3$ and $\beta \geq 2 / 3$, and if one wants to avoid any deviations larger than the $O\left(v_{0}^{-7 / 2}\right)$ term of Eq. (112) at large $v_{0}$, one needs $\alpha \geq 10 / 3$ and $\beta \geq 5 / 6$. The combination of these latter two pairs of inequalities gives what might be called Criterion $\mathrm{B}, \alpha \geq 10 / 3$ and $\beta \geq 5 / 6$.

If one wants a simple choice of the pair of fitting parameters $(\alpha, \beta)$ (e.g., a pair of integers), Criterion A motivated my first choice, $(\alpha, \beta)=(2,1)$. As a crude fit, this is not too bad, giving a maximum relative error of slightly over $10 \%$, e.g. $10.382 \%$ error at $\left(v_{0}, b_{\text {crit }}\left(v_{0}\right)\right)=(0.940172,3.10142)$. My second choice, $(\alpha, \beta)=(3,1)$, is the smallest pair of integers that (almost) satisfies Criterion B. This gives

$$
b_{\text {crit }}\left(v_{0}\right) \simeq B_{(3,1)}\left(v_{0}\right) \equiv\left[\left(6 \pi v_{0}^{-7}\right)^{3 / 5}+8 v_{0}^{-3 / 2}\right]^{1 / 3}+\left[\left(\frac{3125 v_{0}}{216 \pi^{3}}\right)^{1 / 5}+v_{0}^{2}\right]^{-1} .
$$

This approximate formula gives a much better bit, with a maximum relative error of only about $0.85 \%$, near $\left(v_{0}, b_{\text {crit }}\left(v_{0}\right)\right)=(1.79814,1.78528)$. (The position of 
the maximum relative error was not calculated to the six-digit figures given for $\left(v_{0}, b_{\text {crit }}\left(v_{0}\right)\right)$ here, but they are given to six digits so that one can see how accurate this, or some other, approximation is at this sample point, a point where the relative error was about as large as I could find given the six-digit apparent accuracy of the Mathematica calculation.)

I then used the FindMinimum function of Mathematica to minimize, as a function of the pair $(\alpha, \beta)$, the sum of the 100th powers of the relative errors of $B_{(\alpha, \beta)}\left(v_{0}\right)$ over the first 45 pairs of values of $\left(v_{0}, b_{\text {crit }}\left(v_{0}\right)\right)$ that I had calculated. This sum is an analytic function of $(\alpha, \beta)$ whose minimum should be at a value of $(\alpha, \beta)$ which is near the point where the maximum absolute value of the relative error for the 45 points is minimized, since the sum of the 100th power of the relatives errors should be dominated by the largest relative error.

Mathematica gave the minimization as occurring at $(\alpha, \beta)=(4.00448,1.36884)$, though the result would no doubt have been slightly different if I had used a different set of $\left(v_{0}, b_{\text {crit }}\left(v_{0}\right)\right)$ and a different power of the errors. Ideally one should use an infinite set of points and an infinite power of the errors, but I considered 45 points and a power of 100 as being high enough to give a good feel for the true minimum, over $(\alpha, \beta)$, of the maximum of the magnitude of the relative error. The largest relative error I found for this value of $(\alpha, \beta)$ was $0.427328 \%$.

By comparison, minimizing the sum of the squares (2nd powers) of the relative errors led to $(\alpha, \beta)=(3.98412,1.37903)$, which is not that much different even for this relatively small value of the power, though the maximum error I found for this choice of $(\alpha, \beta), 0.47019 \%$, is about $10 \%$ higher.

A fairly simple rational approximation for the $(\alpha, \beta)$ that minimizes the maximum relative error of $B_{(\alpha, \beta)}\left(v_{0}\right)$ is $(4,15 / 11)$, which then leads to

$$
b_{\text {crit }}\left(v_{0}\right) \simeq B_{(4,15 / 11)}\left(v_{0}\right) \equiv\left[\left(\frac{6 \pi}{v_{0}^{7}}\right)^{\frac{4}{5}}+\left(\frac{2}{\sqrt{v_{0}}}\right)^{4}\right]^{\frac{1}{4}}+\left[\left(\frac{3125 v_{0}}{216 \pi^{3}}\right)^{\frac{3}{11}}+v_{0}^{\frac{30}{11}}\right]^{-\frac{11}{15}}
$$

Here the number 4 in the exponents of the first term corresponds to $\alpha$, and the number 11 in the exponents of the second term corresponds to $15 / \beta$, so if one wanted different values of $(\alpha, \beta)$, one could simply change these two numbers in this expression. This approximation gives a maximum relative error of just under $0.43 \%$ (I found a maximum of $0.429991 \%)$, near $\left(v_{0}, b_{\text {crit }}\left(v_{0}\right)\right)=(0.820853,3.54436)$. Thus this $B_{(4,15 / 11)}\left(v_{0}\right)$ is over $99 \%$ as good as $B_{(4.00448,1.36884)}\left(v_{0}\right)$ (has a maximum relative error less than 1.01 times the maximum relative error of $\left.B_{(4.00448,1.36884)}\left(v_{0}\right)\right)$ and is roughly twice as good an approximation (i.e., with roughly half the maximum relative error) as the simpler $B_{(3,1)}\left(v_{0}\right)$ given by Eq. (114) that I guessed on my second attempt without doing any numerical error minimization. To get a relative accuracy better than roughly one part in 234 for $b_{\text {crit }}\left(v_{0}\right)$, one would need a different functional form than $B_{(\alpha, \beta)}\left(v_{0}\right)$ given by Eq. (113), but I won't pursue such refinements here. 
Since the critical impact parameter $b_{\text {crit }}$ goes as $v_{0}^{-7 / 5}$ for small velocity $v_{0}$ and as $v_{0}^{-1 / 2}$ for large $v_{0}$, one can see that if one multiplies $b_{\text {crit }}$ by $v_{0}$ to get the critical initial angular momentum $L_{\text {crit }}\left(v_{0}\right)$ (per unit object mass $M$, and in units of $L_{u}^{2} / T_{u}$ after dividing out by this $M$ ), this rises with both low and high $v_{0}$ and hence must have some minimum in between. By calculating several points near the minimum, I found that the minimum value is

$$
L_{\min } \approx 2.9026
$$

which occurs near the sample point $\left(v_{0}, b_{\text {crit }}\left(v_{0}\right)\right)=(0.834385,3.48671)$ given above. When one restores the units and multiplies by the object mass $M$ to get the true minimum angular momentum of the object about the initial static, straight string configuration, one gets

$$
L_{\min } \approx 2.9026\left[\frac{\pi^{2}}{32} \mu\left(1-\frac{Q^{2}}{M^{2}}\right)^{2}\right]^{1 / 3} M^{2} \approx 1.9611 \mu^{1 / 3}\left(1-Q^{2} / M^{2}\right)^{2 / 3} M^{2}
$$

A compact gravitating (and possibly electrically charged) object approaching from infinity toward an initially static, straight, infinitely long cosmic string with angular momentum (about the initial string axis) less than this would inevitably be captured by the string.

\section{Crude formula for the critical impact parame- ter for all velocities}

In the test-string approximation [3], I calculated the critical impact parameter for velocities much lower than the speed of light and then a correction term linear in the velocity divided by the speed of light, getting Eq. (88) of that paper. In the case of a Schwarzschild black hole $(Q=0)$, I arbitrarily added a (relatively small) constant term to make the resulting formula agree with the known critical impact parameter at the speed of light [1], which is the same as the critical impact parameter for a massless point particle, $\sqrt{27} M$. The resulting formula, Eq. (89) of [3], though just a guess by no means rigorously derived, is apparently a very good approximation to the actual critical impact parameter for a test string at all calculated velocities (i.e., up to a relativistic gamma-factor of $\gamma_{0} \sim 10$ ) [4, 11], though it misses an infinite series of discontinuities that I predicted [3] occur at large values of $\gamma_{0}$ (none seen yet in the analyses of [2, 4] up to $\gamma_{0} \sim 10$ ). At velocities at all comparable to the speed of light, the critical impact parameter apparently must be calculated numerically by solving partial differential equations [2, 4], which is a much more difficult numerical problem than using the ordinary differential equations of [3] and of the present paper that are only accurate at velocities very low compared with the speed of light. 
Here I would like to give an analogue of Eq. (89) of [3] that incorporates (a) Eq. (115) as an excellent approximation to my numerical calculations at all velocities very low compared with the speed of light, (b) Eq. (88) of [3] for the term linear in velocity that is independent of the string tension, and also (c) the right limit for the critical impact parameter at the speed of light for a Reissner-Nordstrom black hole (arbitrary $Q^{2} \leq M^{2}$ ). This last value is the minimum value of $\sqrt{-g_{\theta \theta} / g_{00}}$ in the Reissner-Nordstrom metric, which is

$$
b_{\text {crit }}\left(v_{0}=1\right)=\sqrt{\frac{\left(3 M+\sqrt{9 M^{2}-8 Q^{2}}\right)^{3}}{2 M+2 \sqrt{9 M^{2}-8 Q^{2}}}} .
$$

The resulting crude formula for all velocities for the critical impact parameter for the capture of a Reissner-Nordstrom black hole of mass $M$ and charge $Q$ by an initially straight, static cosmic string with dimensionless tension $\mu \equiv G \mu \ll 1$ is that $b_{\text {crit }}\left(v_{0}\right)$ is roughly the same as $b_{\text {guess }}\left(v_{0}\right)$, with

$$
\begin{aligned}
\frac{b_{\text {guess }}\left(v_{0}\right)}{M} & =\left(\frac{\pi}{2}\right)^{\frac{1}{2}}\left(1-\frac{Q^{2}}{M^{2}}\right)^{\frac{1}{2}} v_{0}^{-\frac{1}{2}}\left(1-v_{0}^{\frac{1}{2}}\right)\left[1+\frac{\pi^{2}}{16}\left(\frac{9}{2}\right)^{\frac{2}{5}}\left(1-\frac{Q^{2}}{M^{2}}\right)^{\frac{6}{5}} \mu^{\frac{12}{5}} v_{0}^{-\frac{18}{5}}\right]^{\frac{1}{4}} \\
& +\frac{\pi}{4}\left(1-\frac{Q^{2}}{M^{2}}\right) \mu v_{0}^{-2}\left(1-v_{0}^{2}\right)\left[1+\left(\frac{3125}{1728}\right)^{\frac{3}{11}}\left(1-\frac{Q^{2}}{M^{2}}\right)^{\frac{9}{11}} \mu^{\frac{18}{11}} v_{0}^{-\frac{27}{11}}\right]^{-\frac{11}{15}} \\
& -\frac{64}{15}\left(1-v_{0}\right)+\sqrt{\frac{\left(3+\sqrt{9-8 Q^{2} / M^{2}}\right)^{3}}{2+2 \sqrt{9-8 Q^{2} / M^{2}}}}
\end{aligned}
$$

The first two terms dominate for $v_{0} \ll 1$ (where we have now returned to units in which the speed of light is $c=1$ ). For this highly nonrelativistic velocity these two terms give essentially the same as Eq. (115), which I found numerically is accurate to greater than $99.5 \%$ accuracy for all nonrelativistic velocities when $\mu \ll 1$. The terms which were added to Eq. (115) to get these two terms are essentially constants (up to correction factors that go to unity when $\mu$ is taken to zero) at $v_{0} \gg \mu^{2 / 3}$ and were designed to make these two terms vanish at $v_{0}=1$. The third term on the right hand side of Eq. (119) is the contribution of the term linear in $v_{0}$ calculated for the test string in [3] (though with the recognition that there might be other such terms missed in that calculation, and perhaps other terms that are independent of $v_{0}$ and that go as $v_{0}^{1 / 2}$ ), corrected with a constant term to make this third term also vanish at $v_{0}=1$. The fourth (and last) term on the right hand side of Eq. (119) is independent of $v_{0}$ and gives the critical impact parameter at the speed of light, the same as that of a massless particle impinging upon the Reissner-Nordstrom black hole.

Although I have no real justification of Eq. (119) for relativistic velocities, the fact that it was found to be a fairly good approximation for all velocities calculated 
so far (i.e., at least for velocities not too ultrarelativistic) for Schwarzschild black holes 11] leads me to conjecture that it may be accurate to within several percent for all velocities for Reissner-Nordstrom black holes as well, assuming only that $\mu \ll 1$. However, apparently only numerical calculations could confirm this.

Following Eq. (90) of [3], one might note that this crude guess, Eq. (119), gives a minimum critical impact parameter of

$$
\frac{b_{\text {guess min }}}{M} \approx 6\left(\frac{\pi}{15}\right)^{\frac{1}{3}}\left(1-\frac{Q^{2}}{M^{2}}\right)^{\frac{1}{3}}-\left(\frac{\pi}{2}\right)^{\frac{1}{2}}\left(1-\frac{Q^{2}}{M^{2}}\right)^{\frac{1}{2}}-\frac{64}{15}+\sqrt{\frac{\left(3+\sqrt{9-8 Q^{2} / M^{2}}\right)^{3}}{2+2 \sqrt{9-8 Q^{2} / M^{2}}}}
$$

at

$$
v_{0} \approx \frac{(225 \pi)^{1 / 3}}{32}\left(1-\frac{Q^{2}}{M^{2}}\right)^{\frac{1}{3}},
$$

ignoring correction terms of order $\mu$ that for $\mu \ll 1$ would be negligible in comparison with the $O(1)$ errors I would expect to be in Eq. (119). It would be interesting to learn from numerical calculations how the minimum critical impact parameter (say divided by $M$ to make it dimensionless), and the velocity at which it occurs, varies as a function of $Q^{2} / M^{2}$ for a Reissner-Nordstrom black hole.

\section{$9 \quad$ Effects of a long string on the sun or earth}

It may be amusing to conclude this hypothetical paper with a discussion of what would happen if a long straight string were impinging upon the sun, earth, or moon.

Approximate these objects as spherical, with mass $M$, charge $Q=0$, and radius $R$, not to be confused with the magnitude of $\mathbf{R}$ defined in Eq. (45). Then the mathematical analysis above applies so long as the string does not get inside the object, so the separation $r$ between the string and the center of mass of the object, the magnitude of $\mathbf{r}$ defined in Eq. (33), must remain greater than $R$. We shall find that for solar system objects, this puts us in the stiff-string regime unless $\mu$ is extremely tiny, much smaller than $\mu \sim 10^{-6}$ expected for cosmic strings.

In the critical capture case, inserting the units $T_{u}$ and $L_{u}$ from Eqs. (35) and (36) into Eqs. (82) and (83), with $L_{0}=b_{\text {crit }} v_{0}$ and with $b_{\text {crit }}\left(v_{0}\right)$ being given by Eq. (81) in this stiff-string regime, gives the minimum separation $r_{m}$ and the relative velocity $v_{m}$ at this separation being given by

$$
\begin{gathered}
r_{m} \approx M\left(\frac{9 \pi^{5}}{2048}\right)^{1 / 5}\left(1-\frac{Q^{2}}{M^{2}}\right)^{3 / 5} \mu^{1 / 5} v_{0}^{-4 / 5} \\
v_{m} \approx\left[\frac{8}{3}\left(1-\frac{Q^{2}}{M^{2}}\right) \mu^{2} v_{0}^{2}\right]^{1 / 5} \approx\left[\frac{\pi \mu}{2}\left(1-\frac{Q^{2}}{M^{2}}\right) \frac{M}{r_{m}}\right]^{1 / 2} .
\end{gathered}
$$


This expression for $v_{m}$ is $\sqrt{\pi \mu / 4}$ times the speed of a test mass, with the same charge-to-mass ratio $Q / M$ as the object, that falls in to the surface of the object from a much lower velocity far away, because the static force given by Eq. (15) between a compact object and a string is $\pi \mu / 4$ times that between the object and a copy of itself at the same separation without the string.

One can now invert Eq. (122) and replace $r_{m}$ by $R$ to get the initial velocity $v_{0}$ needed so that the critical capture occurs with the minimum separation being given by the radius $R$ of the object:

$$
v_{0} \approx\left(\frac{9 \pi^{5}}{2048}\right)^{1 / 4}\left(1-\frac{Q^{2}}{M^{2}}\right)^{3 / 4} \mu^{1 / 4}\left(\frac{M}{R}\right)^{5 / 4} .
$$

The corresponding critical impact parameter is

$$
b_{\text {crit }} \approx M\left(\frac{512}{9 \pi^{3}}\right)^{1 / 4}\left(1-\frac{Q^{2}}{M^{2}}\right)^{-1 / 4} \mu^{1 / 4}\left(\frac{M}{R}\right)^{-7 / 4}
$$

For the initial velocity to obey the criterion $v_{0} \ll\left(1-Q^{2} / M^{2}\right)^{1 / 3} \mu^{2 / 3}$ for the stiffstring approximation to be valid during the critical capture, one needs

$$
\left(1-\frac{Q^{2}}{M^{2}}\right)\left(\frac{M}{R}\right)^{3} \ll \mu \ll 1 .
$$

This is an extremely weak restriction for solar system objects, since $M / R$ is approximately $2.12 \times 10^{-6}$ for the sun, $6.96 \times 10^{-10}$ for the earth, and $3.14 \times 10^{-11}$ for the moon.

For the sun, with mass $M \approx 1.47662504 \mathrm{~km}$, charge $Q \approx 0$, and radius $R \approx$ $6.960 \times 10^{5} \mathrm{~km}$, critical capture with the minimum separation being $R$ gives the initial velocity and impact parameter as $v_{0} \approx 0.827\left(\mu / 10^{-6}\right)^{1 / 4} \mathrm{~m} / \mathrm{s} \approx 2.98\left(\mu / 10^{-6}\right)^{1 / 4}$ $\mathrm{km} / \mathrm{hr}$ and $b_{\text {crit }} \approx 4.61 \times 10^{11}\left(\mu / 10^{-6}\right)^{1 / 4} \mathrm{~m} \approx 3.08\left(\mu / 10^{-6}\right)^{1 / 4} \mathrm{AU}$ (astronomical units). The relative velocity of the sun and string when the string just grazes the sun is $v_{m} \approx 547\left(\mu / 10^{-6}\right)^{1 / 2} \mathrm{~m} / \mathrm{s} \approx 1970\left(\mu / 10^{-6}\right)^{1 / 2} \mathrm{~km} / \mathrm{hr}$. When the string is grazing the sun, it gives the sun an acceleration of $a=\pi \mu M /\left(4 r^{2}\right) \approx 2.15 \times$ $10^{-4}\left(\mu / 10^{-6}\right) \mathrm{m} / \mathrm{s}^{2}$, which is less than the average acceleration of the earth toward the sun, $5.93 \times 10^{-3} \mathrm{~m} / \mathrm{s}^{2}$, unless $\mu \geq 2.76 \times 10^{-5}$. Long slow strings with $\mu \sim 10^{-6}$ or less apparently don't give much danger of pulling the sun away from the earth, but they could change the orbital characteristics of the earth by a few percent, with possibly disastrous climatic effects.

For the earth, with $M \approx 0.444 \mathrm{~cm}, Q \approx 0$, and $R \approx 6371 \mathrm{~km}$, one gets $v_{0} \approx 3.65 \times$ $10^{-5}\left(\mu / 10^{-6}\right)^{1 / 4} \mathrm{~m} / \mathrm{s} \approx 13.1\left(\mu / 10^{-6}\right)^{1 / 4} \mathrm{~cm} / \mathrm{hr}, b_{\text {crit }} \approx 1.73 \times 10^{12}\left(\mu / 10^{-6}\right)^{1 / 4} \mathrm{~m} \approx$ $11.6\left(\mu / 10^{-6}\right)^{1 / 4} \mathrm{AU}$, and $v_{m} \approx 9.91\left(\mu / 10^{-6}\right)^{1 / 2} \mathrm{~m} / \mathrm{s} \approx 35.7\left(\mu / 10^{-6}\right)^{1 / 2} \mathrm{~km} / \mathrm{hr}$, if the earth were isolated so that we could neglect the larger effect of the sun on the earth during the interaction of the string with the earth. When the string is grazing the 
earth, it gives the earth an acceleration of $a \approx 7.71 \times 10^{-6}\left(\mu / 10^{-6}\right) \mathrm{m} / \mathrm{s}^{2}$, which is less than the average acceleration of the earth toward the sun if $\mu<7.68 \times 10^{-4}$. Thus a string with roughly this small a tension or smaller could not kidnap the earth from the sun. The average acceleration of the earth toward the moon is about $3.32 \times 10^{-5} \mathrm{~m} / \mathrm{s}^{2}$, which is greater than the acceleration of the earth toward a string grazing it if $\mu<4.30 \times 10^{-6}$, so a cosmic string with $\mu \sim 10^{-6}$ would be unable to pull the earth away from either the sun or the moon (barring contrived resonance effects), though there is not a large margin of safety for pulling the earth away from the moon.

For the moon, with $M \approx 0.0546 \mathrm{~mm}, Q \approx 0$, and $R \approx 1738 \mathrm{~km}$, one gets $v_{0} \approx$ $7.58 \times 10^{-7}\left(\mu / 10^{-6}\right)^{1 / 4} \mathrm{~m} / \mathrm{s} \approx 2.73\left(\mu / 10^{-6}\right)^{1 / 4} \mathrm{~mm} / \mathrm{hr}, b_{\text {crit }} \approx 5.34 \times 10^{12}\left(\mu / 10^{-6}\right)^{1 / 4} \mathrm{~m}$ $\approx 35.7\left(\mu / 10^{-6}\right)^{1 / 4} \mathrm{AU}$, and $v_{m} \approx 2.10\left(\mu / 10^{-6}\right)^{1 / 2} \mathrm{~m} / \mathrm{s} \approx 7.58\left(\mu / 10^{-6}\right)^{1 / 2} \mathrm{~km} / \mathrm{hr}$, assuming the moon were isolated. At the moon's surface, a string would give it an acceleration of $a \approx 1.27 \times 10^{-6}\left(\mu / 10^{-6}\right) \mathrm{m} / \mathrm{s}^{2}$, which is less than the average acceleration of the moon toward the sun if $\mu<4.66 \times 10^{-3}$, and is less than the average acceleration of the moon toward the earth, $2.70 \times 10^{-3} \mathrm{~m} / \mathrm{s}^{2}$, if $\mu<2.12 \times$ $10^{-3}$. Thus the moon is in even less danger of being pulled away from the sun than the earth is, and even if the earth-moon system were isolated from the sun, a string with reasonably small tension could not remove the moon from the earth.

Another effect one can estimate is that of a string on the tides. If the earth had a string grazing it or running through it, presumably the curvature of spacetime in and near the earth would be altered by a fraction of order $\mu$. The Riemann curvature tensor at the surface of the earth has the orthonormal component $R_{r 0 r}^{0}=2 M / R^{3} \approx 3.08 \times 10^{-6} \mathrm{~s}^{-2} \approx 40.0 \mathrm{hr}^{-2}$, which is about $1.79 \times 10^{7}$ times the corresponding curvature contribution from the moon, and about $3.89 \times 10^{7}$ times the corresponding curvature contribution from the sun. Therefore, if a cosmic string ran through the earth, the perturbation of the earth's tidal force would be of the order of $18\left(\mu / 10^{-6}\right)$ times the tidal force of the moon. If the earth's axis were not aligned with the direction of the string, as the string cuts a swath through the rotating earth, the resulting perturbation in the earth's tidal (curvature) gravitational field might produce Bay-of-Fundy size tides at many coastal cities. Bids are now being accepted for the rights to a disaster movie.

\section{Acknowledgments}

I thank Jean-Pierre De Villiers and Valeri Frolov for getting me interested in this subject, and I thank them and Patrick Brady, Gary Horowitz, and Brandon Carter for further discussions. This work was supported in part by the Natural Sciences and Engineering Research Council of Canada. 


\section{References}

[1] J.-P. De Villiers and V. Frolov, Int. J. Mod. Phys. D7, 957-67 (1998), grqc/9711045.

[2] J.-P. De Villiers and V. Frolov, Phys. Rev. D58, 105018 (1998), gr-qc/9804087.

[3] D. N. Page, Phys. Rev. D58, 105026 (1998), gr-qc/9804088.

[4] J.-P. De Villiers and V. Frolov, "Gravitational Scattering of Cosmic Strings by Nonrotating Black Holes," University of Alberta preprint Thy 08-98 (1998), gr-qc/9812016.

[5] Y. Nambu, in Proceedings of the International Conference on Symmetries \& Quark Models, Wayne State University (1969); Lectures at the Copenhagen Summer Symposium (1970).

[6] T. Goto, Prog. Theor. Phys. 46, 1560 (1971).

[7] B. Carter, Phys. Lett. B224, 61, (1989); J. Geom. Phys. 8, 53 (1992); Class. Quant. Grav. 9, 19 (1992); Phys. Rev. D10, 4835 (1993); in Formation and Interactions of Topological Defects, eds. R. Brandenberger and A.-C. Davis (Plenum, New York, 1995), p. 304, hep-th/9611054; "Brane Dynamics for Treatment of Cosmic Strings and Vortons," Tlaxcala lecture notes, 2nd Mexican School on Gravitation and Mathematical Physics, 1-7 Dec. 1996, eds A. Garcia, C. Lammerzahl, A. Macias, and D. Nunez, hep-th/9705172.

[8] A. Vilenkin, Phys. Rev. D23, 852 (1981).

[9] A. G. Smith, Tufts University Report TUTP-86-11 (1986), published in G. W. Gibbons, S. W. Hawking, and T. Vachaspati, eds., The Formation and Evolution of Cosmic Strings (Cambridge University Press, Cambridge, 1990).

[10] V. P. Frolov, V. D. Skarzhinsky, A. I. Zelnikov, and O. Heinrich, Phys. Lett. B224, 255 (1989); B. Carter and V. P. Frolov, Class. Quant. Grav. 6, 569 (1989).

[11] J. P. De Villiers, private communication.

[12] B. Carter, private communication. 